\title{
Efficient Evaluation of the External Mutual Coupling in Dielectric-Covered Waveguide Slot Arrays
}

\author{
Zusheng Jin, ${ }^{1}$ Giorgio Montisci, ${ }^{2}$ Giovanni Andrea Casula, ${ }^{2}$ Hu Yang, ${ }^{1}$ and Junqi Lu${ }^{1}$ \\ ${ }^{1}$ College of Electronics Science and Engineering, National University of Defense Technology, Changsha 410073, China \\ ${ }^{2}$ Dipartimento di Ingegneria Elettrica ed Elettronica, Università di Cagliari, Piazza D’Armi, 09123 Cagliari, Italy
}

Correspondence should be addressed to Zusheng Jin, jin_hexi@126.com

Received 16 January 2012; Revised 14 April 2012; Accepted 2 May 2012

Academic Editor: Tayeb A. Denidni

Copyright ( 2012 Zusheng Jin et al. This is an open access article distributed under the Creative Commons Attribution License, which permits unrestricted use, distribution, and reproduction in any medium, provided the original work is properly cited.

\begin{abstract}
An accurate and efficient procedure is devised to evaluate the mutual coupling in dielectric-covered planar arrays of longitudinal slots. This approach takes full advantage of the discrete complex image method to cast the spatial-domain Green's functions into closed forms, and hence a direct calculation of mutual coupling in the spatial-domain is available. The computation time reduces significantly compared to the previous spectral-domain procedure, without any loss in the accuracy, rendering this approach very attractive for the design of large dielectric-covered planar arrays.
\end{abstract}

\section{Introduction}

Broadwall waveguide slots are widely employed as radiating elements in arrays for remote sensing, communication, and radar systems up to the millimeter wave frequency band. The advantages of these antennas are the high efficiency, the high gain, a good polarization purity [1], and a considerable mechanical strength.

The analysis and synthesis procedures available in the open literature [2-12] allow to achieve a very good accuracy in the design of waveguide slot arrays. Moreover, the recent advances in manufacturing technology assure high precision in the realization of these antennas even in the millimeter wave range [13]. Different kinds of broadwall radiating slots have been proposed in the past literature: longitudinal slots [1], inclined slots for linear [8], or circular polarization [14], U-shaped slots for circular polarization [15]. Among them, longitudinal slots are the most popular solution, since they allow the higher polarization purity than other waveguide slots. Therefore, their use, started in the 40s, is still continuing nowadays.

The design of planar arrays of longitudinal slots reached a state of maturity with the work by Elliott and his team, and the proposed iterative synthesis procedure has become a standard for the design of these antennas $[1,2]$.
In many practical applications, waveguide slot arrays need to be covered by a dielectric material for protection, aerodynamic purposes, or pressurization of the radiating waveguides $[5,10,16]$. The extension of the Elliott design procedure for such case has been recently proposed by Casula and Montisci [5] for a small planar array.

As it is well known, the external mutual coupling has a significant effect on the performance of a slot array, causing the slots to be detuned at the design frequency. Therefore, an accurate evaluation of the external mutual coupling is required in order to comply with the design specifications on the far-field pattern and on the impedance matching. Moreover, since the iterative synthesis procedure for large arrays is computationally expensive, efficient computation of the external mutual coupling is also required.

In [5], the external mutual coupling is evaluated resorting to the spectral-domain by using the Parseval identity and through numerical integration. A specially tailored numerical technique, the so-called Weighted Average Algorithm (WAA) [17], has been devised, and the integration of the highly oscillatory integrand has been accelerated by using the Shank's transform [18]. However, this approach cannot be considered entirely satisfactory, in particular for the designing of large arrays. Actually, the convergence of the integral in the mutual coupling expression deteriorates rapidly as 
the distance between the slots increases. As a result, its evaluation becomes time-consuming, and it is hard to ensure the accuracy for large distances. Moreover, the WAA requires to extensively investigate the behavior of the integrand, rendering the spectral-domain approach inefficient and not robust. In fact, the oscillation period depends in a complicated manner on the spacing between the slots, and the WAA must be tailored to this behavior.

These drawbacks can be avoided if the calculation is performed directly in the spatial-domain, which is the aim of this paper. An alternative spatial-domain approach for the computation of the external mutual coupling has been proposed with the help of the discrete complex image method (DCIM). Numerical examples show that the computational time required for an accurate evaluation of the mutual coupling is only a small fraction of that required by the previous spectral-domain procedure. Moreover, the computational time and the accuracy are independent of the distance between the slots, and no special treatments are needed for large distances, as required by the procedure presented in [5].

The spatial-domain approach is described in detail in Section 2. Section 3 provides several numerical examples to validate the efficiency and accuracy of this procedure, as compared to the spectral-domain approach of [5].

\section{Evaluation of the External Mutual Coupling}

The external mutual coupling between two arbitrary slots in a dielectric-covered planar array, as depicted in Figure 1, is proportional to the reaction integral between the magnetic field of one slot (slot $n$ ) and the equivalent current distribution of another (slot $m$ ) [2] which can be represented as

$$
g_{n m}=A \int_{\text {slot } m} \underline{H}_{d}\left(\underline{M}_{n}\right) \cdot \underline{M}_{m} d S,
$$

where $A$ is a suitable normalization coefficient; $\underline{M}_{m}$ and $\underline{M}_{n}$ are the normalized equivalent magnetic currents on the slot $m$ and $n$, respectively; $\underline{H}_{d}$ is the magnetic field in the dielectric cover due to the equivalent magnetic current $\underline{M}_{n}$ and can be expressed through the vector potential $\underline{F}_{d}$ :

$$
\underline{H}_{d}\left(\underline{M}_{n}\right)=-j \omega \varepsilon \underline{F}_{d}\left(\underline{M}_{n}\right)+\frac{1}{j \omega \mu} \nabla \nabla \cdot \underline{F}_{d}\left(\underline{M}_{n}\right),
$$

wherein $\omega$ is the angular frequency; $\varepsilon$ and $\mu$ denote the permittivity and permeability of the dielectric cover, respectively.

Substituting (2) into (1), after an integration by parts of the second term, we obtain

$$
g_{n m}=A\left(-j \omega \varepsilon I_{1, n m}+\frac{1}{j \omega \mu} I_{2, n m}\right)
$$

with

$$
\begin{aligned}
& I_{1, n m}=\int_{\text {slot } m} \underline{F}_{d}\left(\underline{M}_{n}\right) \cdot \underline{M}_{m} d S, \\
& I_{2, n m}=-\int_{\text {slot } m} \nabla \cdot \underline{F}_{d}\left(\underline{M}_{n}\right) \nabla \cdot \underline{M}_{m} d S .
\end{aligned}
$$

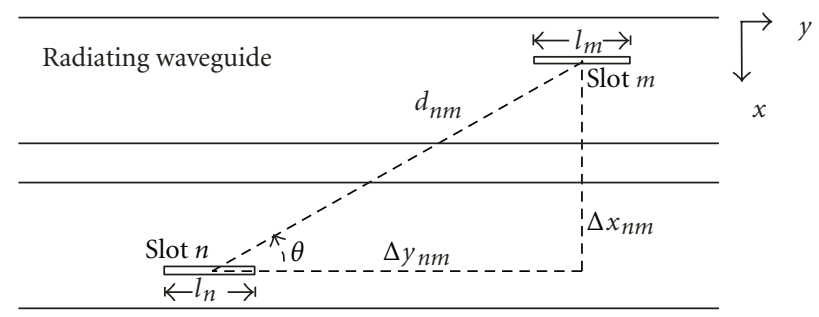

(a)

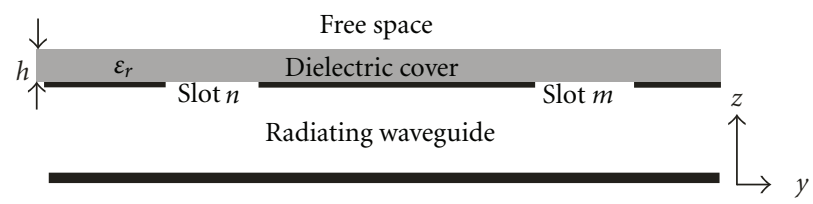

(b)

FIgURE 1: Two arbitrary slots in a planar array (a) top view and (b) sectional view.

Using the well-known assumption of a monomodal electric field distribution on a longitudinal slot [2], we get

$$
\begin{aligned}
I_{1, n m}= & V_{n}^{s} V_{m}^{s} \\
& \times \int_{-l_{m} / 2}^{l_{m} / 2} \int_{-l_{n} / 2}^{l_{n} / 2} G\left(\rho_{n m}\right) \cos \left(\frac{\pi y_{n}}{l_{n}}\right) \\
& \times \cos \left(\frac{\pi y_{m}}{l_{m}}\right) d y_{n} d y_{m}, \\
I_{2, n m}= & \frac{\pi V_{n}^{s} V_{m}^{s}}{l_{m}} \\
& \times \int_{-l_{m} / 2}^{l_{m} / 2} \int_{-l_{n} / 2}^{l_{n} / 2} \nabla \cdot\left[G\left(\rho_{n m}\right) \cos \left(\frac{\pi y_{n}}{l_{n}}\right)\right] \\
& \times \sin \left(\frac{\pi y_{m}}{l_{m}}\right) d y_{n} d y_{m},
\end{aligned}
$$

wherein $V_{n}^{s}$ and $V_{m}^{s}$ are the slot voltages; $l_{n}$ and $l_{m}$ are the slot lengths (Figure 1); $G(\rho)$ is the Green's function of $\underline{F}_{d}$, where $\rho_{n m}$ denotes the distance between the source point and the field point. Once the spatial-domain Green's function $G(\rho)$ has been obtained, the double integrals in (6) can easily be solved via a Gaussian quadrature.

The spatial-domain Green's functions can be obtained from their spectral-domain counterparts by the following Sommerfeld integral

$$
G(\rho)=\frac{1}{4 \pi} \int_{-\infty}^{\infty} d k_{\rho} k_{\rho} H_{0}^{(2)}\left(k_{\rho} \rho\right) \tilde{G}\left(k_{\rho}\right),
$$

where $H_{0}^{(2)}$ is the zero-order-second-kind Hankel function, and $\widetilde{G}\left(k_{\rho}\right)$ is the spectral-domain Green's function, which can be obtained analytically both in the free space and in planar media [19]. For the free-space case, $\tilde{G}\left(k_{\rho}\right)=j \exp \left(-j k_{z 0} z\right) /$ $k_{z 0}$ with $k_{z 0}^{2}=k_{0}^{2}-k_{\rho}^{2}$, and therefore $G(\rho)=\exp \left(-j k_{0} r\right) /$ $(2 \pi r)$ with $r^{2}=\rho^{2}+z^{2}$. For the dielectric-covered case, $\widetilde{G}\left(k_{\rho}\right)$ is given by

$$
\tilde{G}\left(k_{\rho}\right)=\frac{1+j \varepsilon_{r}\left(k_{z 0} / k_{z 1}\right) \tan \left(k_{z 1} h\right)}{D_{\mathrm{TM}}}
$$


with $D_{\mathrm{TM}}=-j \varepsilon_{r} k_{z 0}+k_{z 1} \tan \left(k_{z 1} h\right), k_{z i}^{2}=k_{i}^{2}-k_{\rho}^{2}, i=0,1$, where $\varepsilon_{r}$ is the relative permittivity of the dielectric cover, and $h$ is the dielectric thickness. The constants $k_{0}$ and $k_{1}$ are the wave numbers of the free space and of the dielectric medium, respectively. In this case, integrals (6) cannot be solved in closed form, and the direct numerical integration of the Sommerfeld integral is very time-consuming due to the highly oscillating and slow converging nature of its integrand.

In order to avoid the time-consuming integration typical of the spectral-domain approach, in this work, the computation of the mutual coupling is performed in the spatialdomain by means of a three-level DCIM [20].

The DCIM was first proposed by Fang et al. in 1988 [21] and is under development till now [22-24]. The basic idea of DCIM is to approximate the spectral-domain Green's functions in terms of complex exponentials and then transform them into the spatial-domain analytically with the help of the Sommerfeld identity.

Before the implementation of the approximation, the surface wave poles (SWPs) must be extracted from $\widetilde{G}\left(k_{\rho}\right)$ and their contribution will be analytically added after the approximation. This extraction is necessary in order to ensure the accuracy when the field point is far from the source point. Since the spectral function is an even function of $k_{\rho}$, the poles occur in complex conjugate pairs and the surface wave term in the spectral-domain can be represented mathematically as

$$
\widetilde{G}_{\mathrm{sw}}\left(k_{\rho}\right)=\sum_{i} \frac{2 k_{\rho i} \operatorname{Res}_{i}}{k_{\rho}^{2}-k_{\rho i}^{2}},
$$

where $k_{\rho i}$ and Res $s_{i}$ are the $i$ th SWP and the corresponding residue. Substituting (9) into (7), the SWP contributions are obtained as

$$
G_{\mathrm{sw}}(\rho)=-\frac{j}{2} \sum_{i} H_{0}^{(2)}\left(k_{\rho i} \rho\right) k_{\rho i} \operatorname{Res}_{i}
$$

The remaining part $\widetilde{G}\left(k_{\rho}\right)-\widetilde{G}_{s \mathrm{w}}\left(k_{\rho}\right)$, denoted as $\widetilde{G}_{1}\left(k_{\rho}\right)$, can be approximated by complex exponentials via a threelevel generalized pencil of function (GPOF) method [20, 25]. The so-called three-level GPOF, an extension of the previous two-level version, is capable of approximating the lateral waves by introducing an additional path around the branch point. There may be many different parameterizations of the paths, and the one applied here, first proposed and demonstrated by Alparslan et al. [20, Equation 13], provides very accurate closed-form Green's functions over all ranges of distances.

By performing the three-level GPOF, $\widetilde{G}_{1}\left(k_{\rho}\right)$ can be approximated in terms of complex exponentials. Then, using the well-known Sommerfeld identity, the spatial-domain counterpart of $\widetilde{G}_{1}\left(k_{\rho}\right)$, denoted as $G_{1}(\rho)$, can be written as

$$
G_{1}(\rho)=\frac{1}{2 \pi} \sum_{q=1}^{3} \sum_{p=1}^{P_{q}} A_{p q} \frac{e^{-j k_{0} r_{p q}}}{r_{p q}},
$$

where $r_{p q}=\sqrt{\rho^{2}-B_{p q}^{2}}$ is a complex function of $\rho$. The complex coefficients $A_{p q}$ and the complex exponents $B_{p q}$

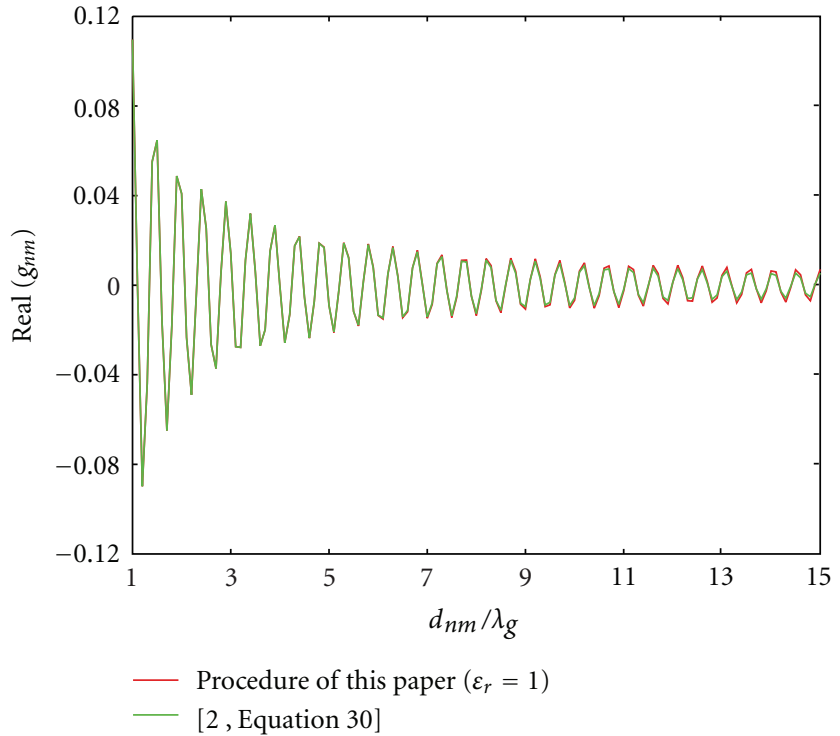

Figure 2: Real part of the mutual coupling coefficient $g_{n m}, \theta=45^{\circ}$.

are obtained through the GPOF procedure; $P_{q}$ denotes the number of exponentials used in the approximation in the $q$ th level.

Finally, combining all the approximating exponentials, together with the SWP contributions, the spatial-domain Green's function can be expressed in closed form $G(\rho)=$ $G_{\text {sw }}(\rho)+G_{1}(\rho)$.

Once the spatial-domain Green's function is available, the computation of the mutual coupling (6) is straightforward. A good accuracy is expected for all slots spacings, since the spatial-domain Green's functions can be calculated accurately in all ranges via the three-level DCIM [20]. Moreover, the coefficients and exponents in the closed-form expression of the Green's function need to be generated for only once, leading to a very efficient calculation.

\section{Results}

In all the simulations presented in this section, we consider two longitudinal slots with lengths $l_{n}=l_{m}=15.5 \mathrm{~mm}$ on a standard WR90 waveguide $(22.86 \mathrm{~mm} \times 10.16 \mathrm{~mm})$. Let $\lambda_{g}$ be the guided wavelength of the radiating guide fundamental mode and $\theta$ the angle between the waveguide axis and the line connecting the centers of the slots (Figure 1).

In order to assess the procedure of this paper, first of all, we test its accuracy against the expression of the mutual coupling obtained by Elliott in of [2, Equation 30] for an array radiating in free space, that is, for $\varepsilon_{r}=1$ (see Figure 1). The operating frequency is $9 \mathrm{GHz}$ and $\theta=45^{\circ}$. The results are reported in Figures 2 and 3, where we show the mutual coupling coefficient as a function of the normalized distance between the slots $d_{n m} / \lambda_{g}$. An excellent agreement can be observed.

Then, the spatial-domain procedure has been compared against the spectral-domain approach, which has been validated for a small array in [5]. A number of simulations have 


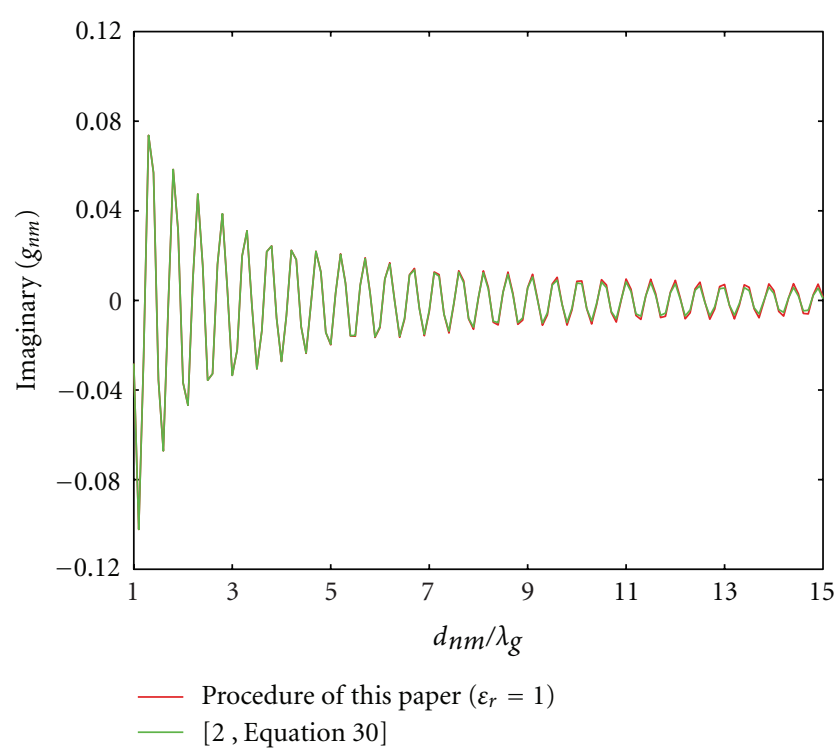

FIGURE 3: Imaginary part of the mutual coupling coefficient $g_{n m}$, $\theta=45^{\circ}$.

been performed (on an Intel Core I7 CPU at $2.80 \mathrm{GHz}$ with $8 \mathrm{~GB}$ RAM) at the operating frequency of $9.4 \mathrm{GHz}$. The dielectric cover thickness is $h=0.165 \mathrm{~mm}$, and its permittivity is $\varepsilon_{r}=2.05$.

In Figures 4 and 5, we show the comparison between the spatial-domain procedure proposed in this paper and the procedure used in [5] for $\theta=0$. The curves have been divided in two figures with different scales in order to better show the behavior of the mutual coupling for large values of $d_{n m}$. The mutual coupling coefficient computed either with the DCIM-based procedure or with the spectral-domain approach of [5] virtually overlap until $d_{n m}=12.5 \lambda_{g}$. For larger values, the results of the procedure used in [5] are not satisfactory, according to what we have pointed out in the Introduction.

For small values of $\theta$ (near zero), the results of the comparison are similar to those of Figures 4 and 5, though the convergence of [5, Equation 6] is achieved for a slightly larger value of the maximum distance between the slots. In Figures 6 and 7, we show the comparison for $\theta=45^{\circ}$. In this case, a good agreement can be observed until $d_{n m}=16 \lambda_{g}$. For $\theta$ around $90^{\circ}$, the procedure of [5] is more stable. In Figures 8 and 9 , the comparison for $\theta=90^{\circ}$ is shown: in this case, the results of the procedure in [5] are accurate enough until $d_{n m}$ around $18 \lambda_{g}$.

As apparent in Figures 4-9, the deterioration of the mutual coupling coefficient computed with the spectraldomain approach of [5] is mainly due to the contribution of its real part.

In [5], the mutual coupling coefficient has been expressed in the spectral-domain by means of the Parseval identity and the resulting integral has been evaluated by dividing the integration domain into three intervals (see [5, Section 2]). We have found that the integral in the unbounded region, wherein the integrand is highly oscillatory and convergence

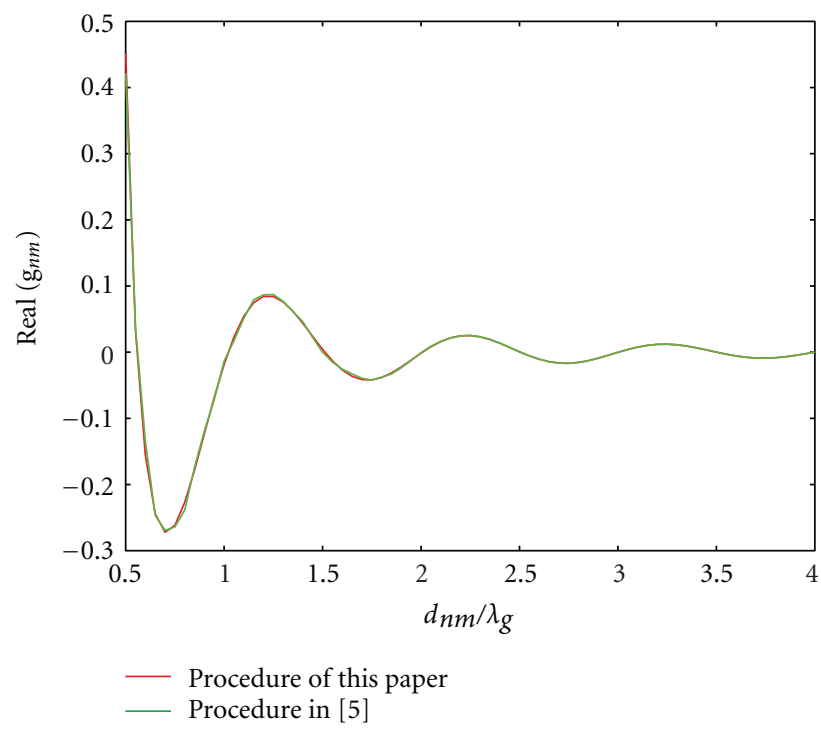

(a)

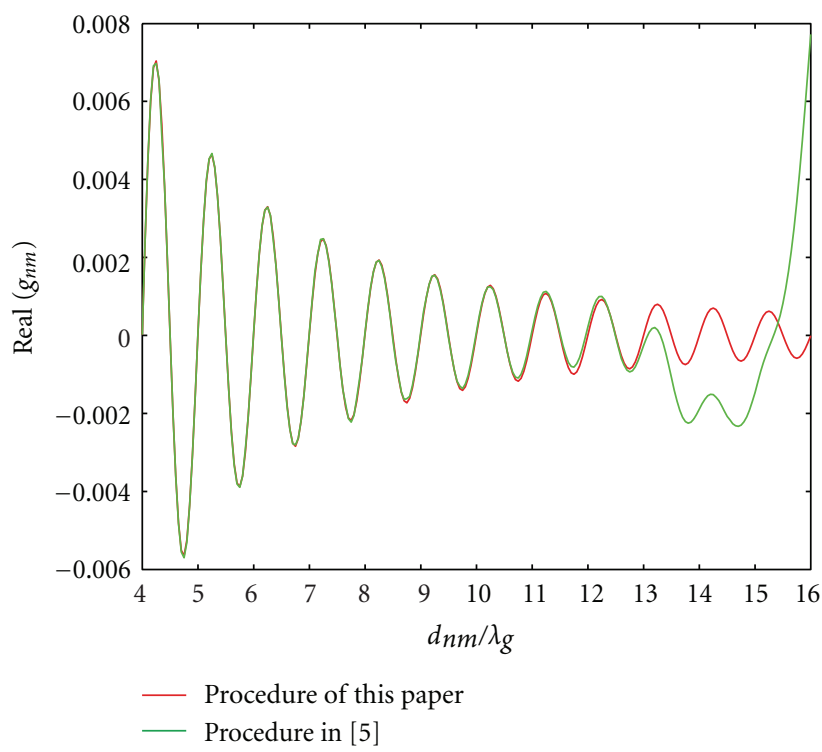

(b)

FIgURE 4: Real part of the mutual coupling coefficient $g_{n m}, \theta=0$.

is very slow, is always real, and this is the reason for the strong instability of the real part of the mutual coupling coefficient.

The proposed spatial-domain procedure has been tested and compared with the spectral-domain approach of [5] also for different dielectric cover permittivity and thickness. The same behavior as for the case investigated above has been observed.

We have found that the DCIM-based spatial-domain approach is not only stable but also efficient. Actually, the convergence of the results is assured regardless both of the distance between the slots $d_{n m}$ and of the angle $\theta$. Here are the parameters of the DCIM for the related simulations presented in this section: $T_{01}=200, T_{02}=2, T_{03}=99, P_{1}=$ $6, P_{2}=2, P_{3}=2$, and $N_{1}=200, N_{2}=100, N_{3}=100$. $P_{q}(q=1,2,3)$ denotes the number of exponentials used 


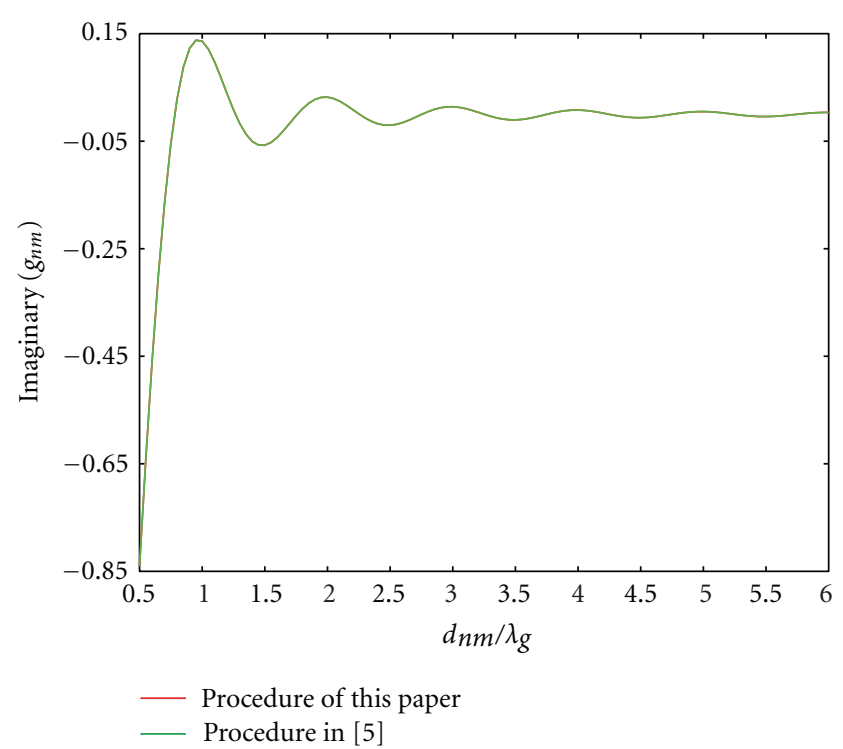

(a)

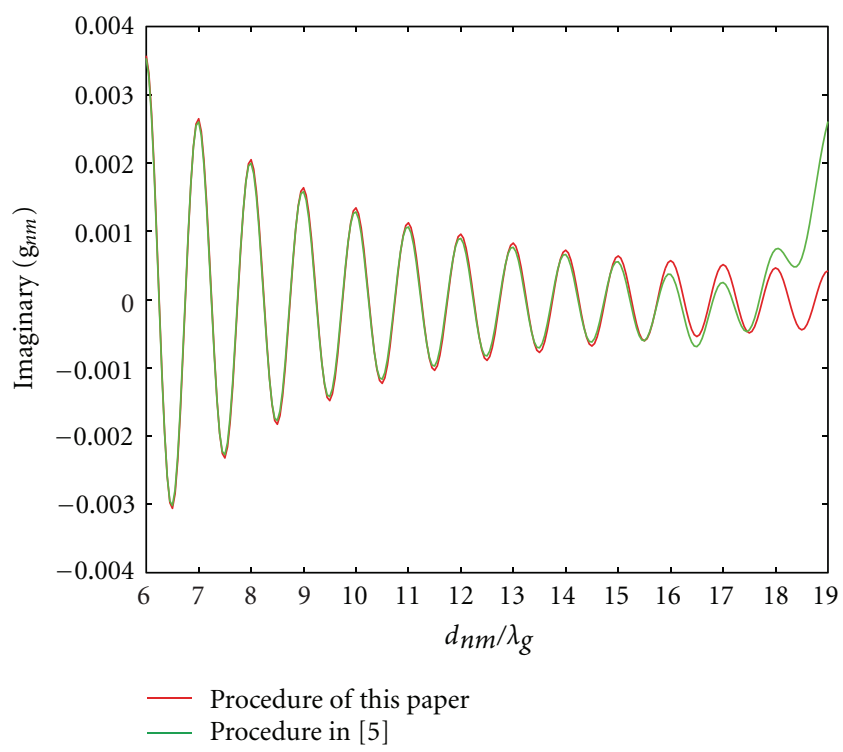

(b)

FIGURE 5: Imaginary part of the mutual coupling coefficient $g_{n m}$, $\theta=0$.

in the approximation in the qth level and is defined in (11). According to [20], $T_{01}, T_{02}$, and $T_{03}$ are the truncation points for the parametrization of the paths $\left(C_{\mathrm{ap} 1}, C_{\mathrm{ap} 2}\right.$, and $\left.C_{\mathrm{ap} 3}\right)$; $N_{1}, N_{2}$, and $N_{3}$ are the number of samples of the GPOF approximation in the paths $\left(C_{\mathrm{ap} 1}, C_{\mathrm{ap} 2}\right.$, and $\left.C_{\mathrm{ap} 3}\right)$, respectively. With this choice, the spatial-domain procedure takes about 0.05 seconds to compute the mutual coupling coefficient, and, as pointed out before, this computational time does not depend on the relative position between the slots.

On the other hand, if the mutual coupling is calculated in the spectral-domain by using [5, Equation 6], the integration parameters strongly depend on $d_{n m}$ and $\theta$. According to [5], we have selected here the parameters of the WAA in order

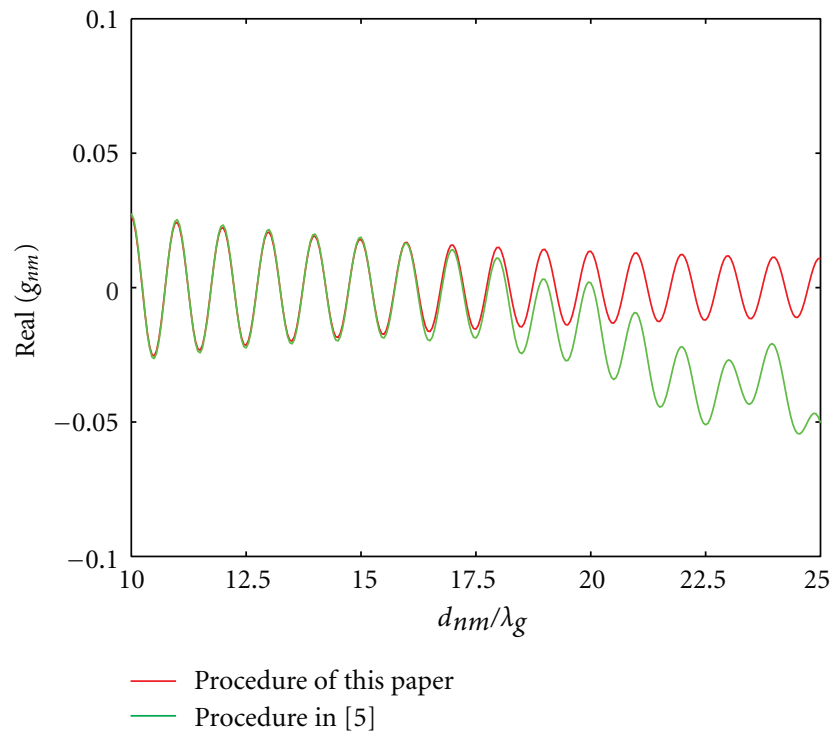

Figure 6: Real part of the mutual coupling coefficient $g_{n m}, \theta=45^{\circ}$. The results for $d_{n m}<10 \lambda_{g}$ are not shown since the curves virtually overlap.

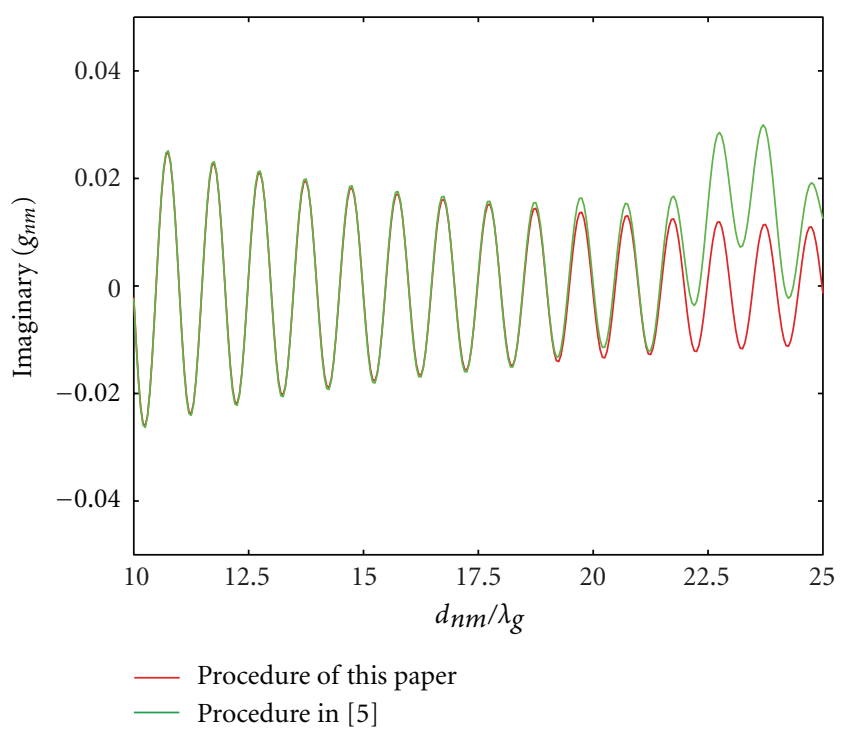

FIGURE 7: Imaginary part of the mutual coupling coefficient $g_{n m}$, $\theta=45^{\circ}$. The results for $d_{n m}<10 \lambda_{g}$ are not shown since the curves virtually overlap.

to obtain the convergence of the integrations for each $\theta$ and a maximum value of $d_{n m}$ equal to $12 \lambda_{g}$. In particular, 15 iterations have been used in the WAA. In this case, the computational time required for the evaluation of the mutual coupling is always about tens of times slower than the spatialdomain procedure. If a $d_{n m}$ larger than $12 \lambda_{g}$ is required, in order to achieve a good accuracy, the integration parameters in the spectral-domain approach of [5] must be suitably recomputed. However, since the convergence of the integral in the mutual coupling expression deteriorates rapidly as 


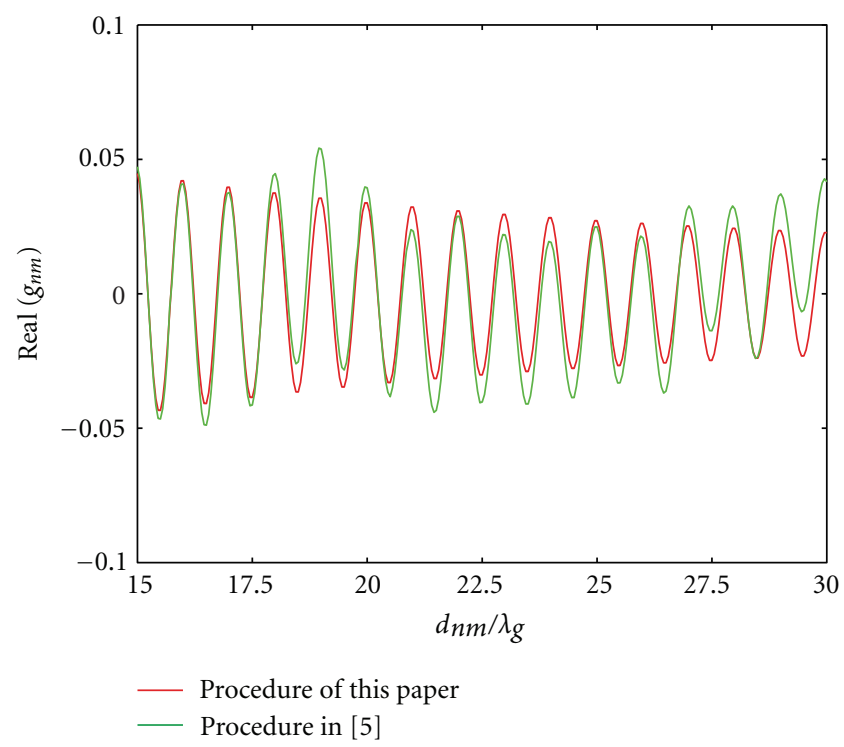

FIGURE 8: Real part of the mutual coupling coefficient $g_{n m}, \theta=90^{\circ}$. The results for $d_{n m}<15 \lambda_{g}$ are not shown since the curves virtually overlap.

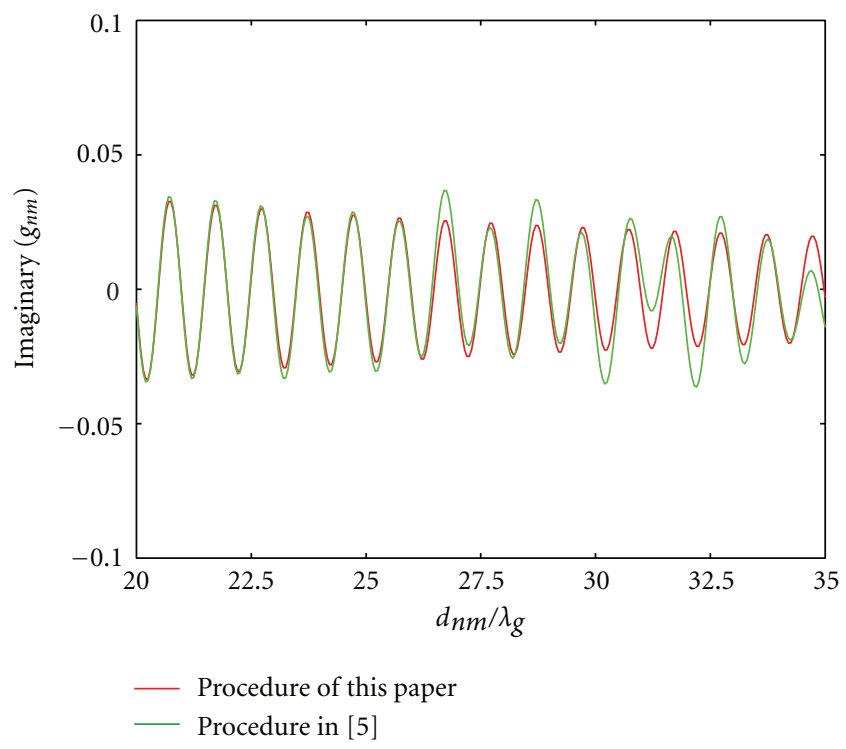

FIGURE 9: Imaginary part of the mutual coupling coefficient $g_{n m}$, $\theta=90^{\circ}$. The results for $d_{n m}<20 \lambda_{g}$ are not shown since the curves virtually overlap.

the distance between the slots increases, in this case, its evaluation becomes very time-consuming.

To rate the improvements in the computational time of the approach proposed in this paper, we take a general $\theta=0$ case (Figures 4 and 5 ) as an example. The results are shown in Table 1. The larger the maximum distance between two arbitrary slots, the larger the computational time required for the convergence of the integration technique of [5]. On the contrary, the approach presented in this paper, based on the DCIM, takes about 0.05 seconds to compute the mutual coupling with the same accuracy of [5], and this time is
TABLE 1: Comparison between the computational times needed for the evaluation of the mutual coupling, $\theta=0$.

\begin{tabular}{lcc}
\hline$d_{n m} / \lambda_{g}$ & Procedure in [5] & Procedure of this paper \\
\hline 4 & $2.1 \mathrm{sec}$ & $0.05 \mathrm{sec}$ \\
6 & $3.2 \mathrm{sec}$ & $0.05 \mathrm{sec}$ \\
8 & $4.2 \mathrm{sec}$ & $0.05 \mathrm{sec}$ \\
10 & $5.3 \mathrm{sec}$ & $0.05 \mathrm{sec}$ \\
12 & $6.3 \mathrm{sec}$ & $0.05 \mathrm{sec}$ \\
\hline
\end{tabular}

independent of the distance between the slots. Therefore, the technique proposed in this paper is significantly faster than that of [5] and the improvement increases for larger arrays.

\section{Conclusion}

A spatial-domain approach has been proposed to evaluate the external mutual coupling in dielectric-covered waveguide slot planar arrays. The presented procedure is based on the use of the DCIM for the approximation of the spatialdomain Green's function. The computational time and the accuracy are independent of the distance between the slots, leading to an accurate and efficient evaluation for all ranges of distances. Numerical examples show that the computational time reduces significantly with respect to the spectraldomain approach without any loss in the accuracy.

\section{Acknowledgments}

The authors would like to express their appreciation to Ye Liangfeng of NUDT, Antenna Research Center, and to Professor G. Mazzarella for helpful discussion and suggestions throughout the study. The authors also wish to acknowledge Professor Z. H. Firouzeh of Amirkabir University of Technology for his instructive discussion pertaining to GPOF, and Xi He of Hangzhou Normal University for her instructive suggestions.

\section{References}

[1] R. S. Elliott, Antenna Theory and Design, Prentice-Hall, New York, NY, USA, 1981.

[2] R. S. Elliott, "An improved design procedure for small arrays of shunt slots," IEEE Transactions on Antennas and Propagation, vol. 31, no. 1, pp. 48-53, 1983.

[3] G. Montisci, "Design of circularly polarized waveguide slot linear arrays," IEEE Transactions on Antennas and Propagation, vol. 54, no. 10, pp. 3025-3029, 2006.

[4] G. A. Casula and G. Mazzarella, "Design of slot arrays in waveguide partially filled with dielectric slab," Electronics Letters, vol. 42, no. 13, pp. 730-731, 2006.

[5] G. A. Casula and G. Montisci, "Design of dielectric-covered planar arrays of longitudinal slots," IEEE Antennas and Wireless Propagation Letters, vol. 8, pp. 752-755, 2009.

[6] S. Costanzo, G. A. Casula, A. Borgia et al., "Synthesis of slot arrays on integrated waveguides," IEEE Antennas and Wireless Propagation Letters, vol. 9, pp. 962-965, 2010.

[7] T. Suzuki, J. Hirokawa, and M. Ando, "Iteration-free design of waveguide slot array with cavities," IEEE Transactions on 
Antennas and Propagation, vol. 58, no. 12, pp. 3891-3897, 2010.

[8] G. Mazzarella and G. Montisci, "Full-wave analysis of dielectric-covered radiating series slots," Microwave and Optical Technology Letters, vol. 20, no. 1, pp. 67-72, 1999.

[9] M. Mondal and A. Chakraborty, "Resonant length calculation and radiation pattern synthesis of longitudinal slot antenna in rectangular waveguide," Progress in Electromagnetics Research Letters, vol. 3, pp. 187-195, 2008.

[10] G. Mazzarella and G. Montisci, "Rigorous analysis of dielectric-covered narrow longitudinal shunt slots with finite wall thickness," Electromagnetics, vol. 19, no. 5, pp. 407-418, 1999.

[11] G. Montisci and G. Mazzarella, "Full-wave analysis of a waveguide printed slot," IEEE Transactions on Antennas and Propagation, vol. 52, no. 8, pp. 2168-2171, 2004.

[12] G. Mazzarella and G. Montisci, "Wideband equivalent circuit of a centered-inclined waveguide slot coupler," Journal of Electromagnetic Waves and Applications, vol. 14, no. 1, pp. 133151, 2000.

[13] Y. Miura, J. Hirokawa, M. Ando, Y. Shibuya, and G. Yoshida, "Bandwidth of a four-aperture element for a double-layer corporate-feed hollow-waveguide slot array," IEEE Transactions on Antennas and Propagation, vol. 59, pp. 2844-2851, 2011.

[14] G. Montisci, M. Musa, and G. Mazzarella, "Waveguide slot antennas for circularly polarized radiated field," IEEE Transactions on Antennas and Propagation, vol. 52, no. 2, pp. 619-623, 2004.

[15] K. S. Min, J. Hirokawa, M. Ando, and N. Goto, "U-shaped slots for circularly polarized slotted waveguide array," in Proceedings of the IEEE Antennas and Propagation Society International Symposium, pp. 1434-1437, Digest, June 1995.

[16] P. B. Katehi, "Dielectric-covered waveguide longitudinal slots with finite wall thickness," IEEE Transactions on Antennas and Propagation, vol. 38, no. 7, pp. 1039-1045, 1990.

[17] T. Itoh, Numerical Techniques for Microwave and MillimeterWave Passive Structures, Wiley, New York, NY, USA, 1989.

[18] A. Alaylioglu, G. A. Evans, and J. Hyslop, "The evaluation of oscillatory integrals with infinite limits," Journal of Computational Physics, vol. 13, no. 3, pp. 433-438, 1973.

[19] K. A. Michalski and J. R. Mosig, "Multilayered media Green's functions in integral equation formulations," IEEE Transactions on Antennas and Propagation, vol. 45, no. 3, pp. 508-519, 1997.

[20] A. Alparslan, M. I. Aksun, and K. A. Michalski, "Closedform Green's functions in planar layered media for all ranges and materials," IEEE Transactions on Microwave Theory and Techniques, vol. 58, no. 3, pp. 602-613, 2010.

[21] D. G. Fang, J. J. Yang, and G. Y. Delisle, "Discrete image theory for horizontal electric dipoles in a multilayered medium," Proceedings of the IEEE, vol. 135, no. 5, pp. 297-303, 1988.

[22] M. I. Aksun and G. Dural, "Clarification of issues on the closed-form Green's functions in stratified media," IEEE Transactions on Antennas and Propagation, vol. 53, no. 11, pp. 3644-3653, 2005.

[23] M. Yuan, T. K. Sarkar, and M. Salazar-Palma, "A direct discrete complex image method from the closed-form Green's functions in multilayered media," IEEE Transactions on Microwave Theory and Techniques, vol. 54, no. 3, pp. 1025-1032, 2006.

[24] L. Zhuang, G. Zhu, Y. Zhang, and B. Xiao, "An improved discrete complex image method for Green's functions in multilayered media," Microwave and Optical Technology Letters, vol. 49, no. 6, pp. 1337-1340, 2007.
[25] Y. Hua and T. K. Sarkar, "Generalized pencil-of-function method for extracting poles of an EM system from its transient response," IEEE Transactions on Antennas and Propagation, vol. 37, no. 2, pp. 229-234, 1989. 

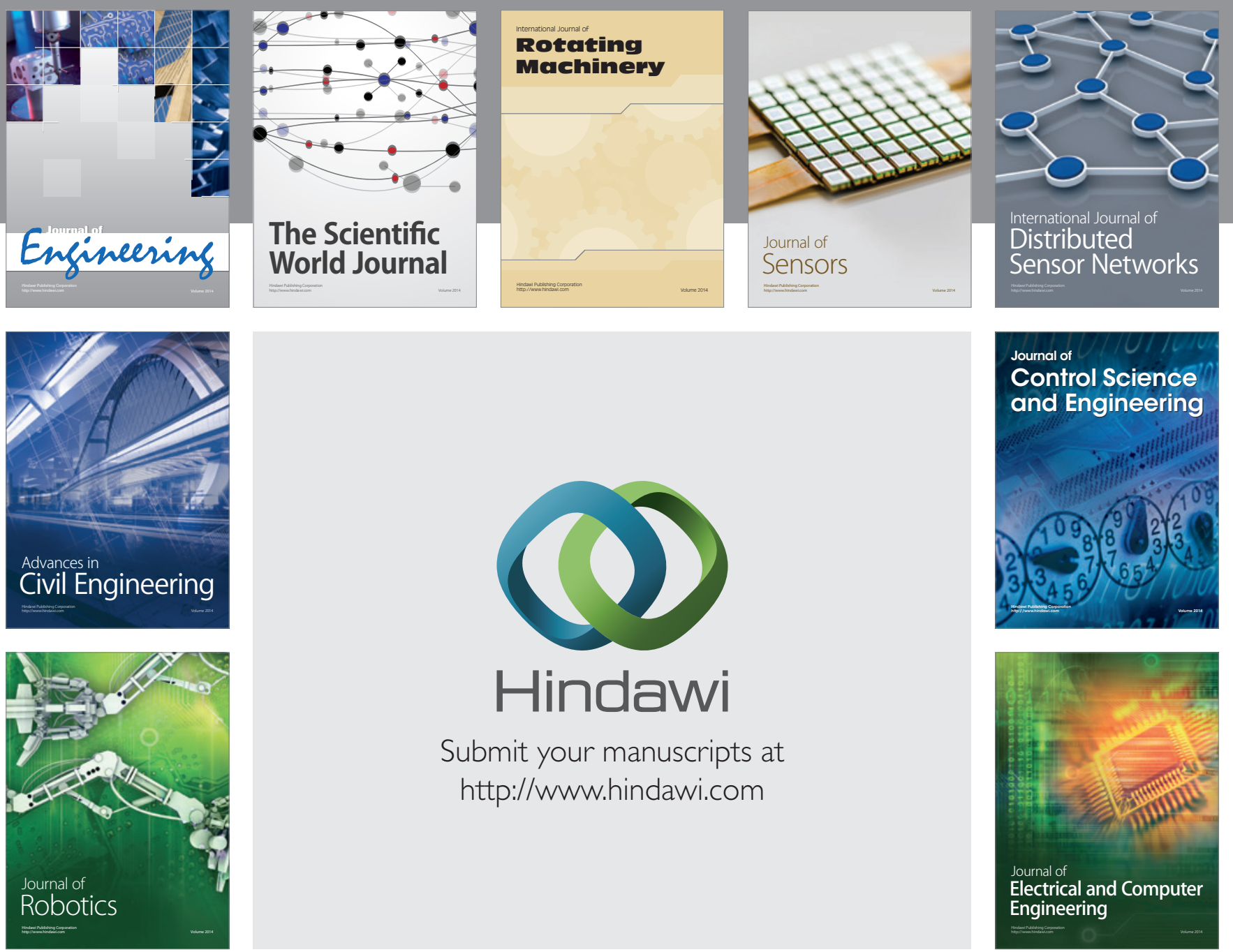

Submit your manuscripts at

http://www.hindawi.com
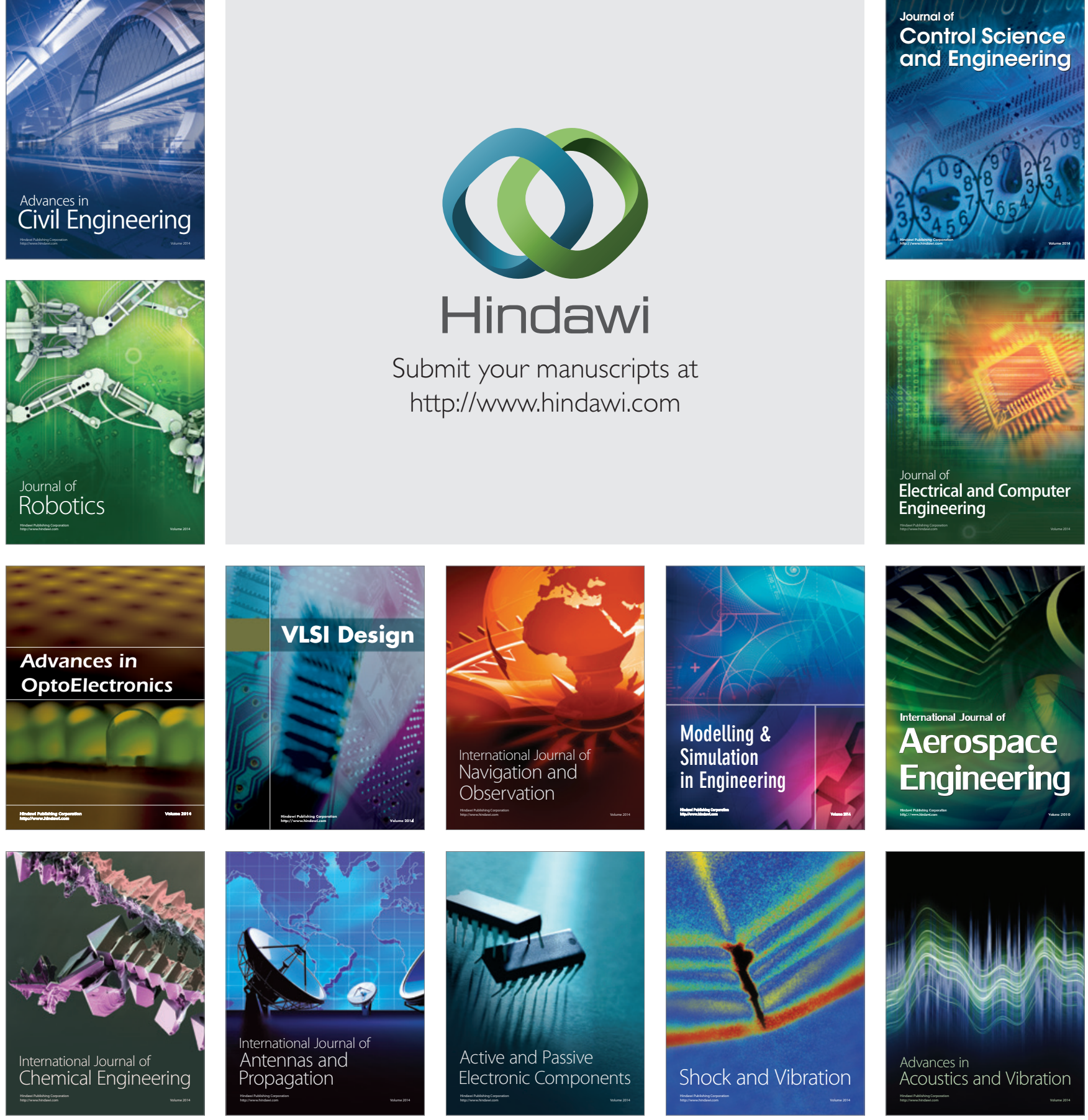